\section{Leadership in healthcare}

\section{DEVELOPING EFFECTIVE LEADERS: CREATING CHANGE CHAMPIONS IN CHILDREN'S HEALTHCARE}

${ }^{1}$ Emma Parish*, ${ }^{2}$ Jane Runnacles, ${ }^{3}$ Nicola Davey, ${ }^{4}$ Alice Roueché. ${ }^{1}$ Homerton University Hospital, London, UK; ${ }^{2}$ Royal Free Hospital, London, UK; ${ }^{3}$ Quality Improvement Clinic, UK; ${ }^{4}$ Evelina London Children's Hospital, London, UK

\subsection{6/leader-2018-FMLM. 100}

Aim Our aim is to equip those working with young people across healthcare in London with the skills and habits of an improver, enhance local services, and become role models for Quality Improvement (QI).

Methods The London School of Paediatrics (LSP) worked with QIClearn $^{\mathrm{TM}}$ to deliver a blended learning programme. An initial pilot in 2017 showed that many attending lacked understanding of QI (35\% stating they had never seen the QI Model for Improvement). Post-pilot feedback was positive $(83 \%$ felt empowered that they can make changes within their workplace, $80 \%$ had taught someone else something about QI).

Building on this feedback, in 2018, we delivered a 16 week course to 23 participants (18 trainees, 1 pharmacist, 1 consultant and 3 patient and carer learners), taking them through the Model for Improvement, measurement, human factors and presentation. A bespoke online social learning platform was developed. Participants worked through content with the support of a mentor, while carrying out QI projects in their workplace. Interactive modules, resources, forums and webinars were complemented by 2 full-day face-to-face workshops. We used QI methods to assess and improve the programme.

Results Initially $50 \%$ of participants stated it was the first time they had seen the Model of Improvement and only 1 in 5 had used it and felt confident. At the second workshop everyone had seen the Model and 50\% had used it and felt confident. All participants who started the programme were awarded certificates of completion, 8 were recognised as Change Champions (showing highly improved knowledge and completion of a project to presentation), one of the attendees has been identified as a mentor-in-training for the next cohort. Further data is being collected on sustainable practice. Conclusion This programme shows that blended learning with mentoring can support the professional development of emerging leaders within QI.

\section{Leading innovation and improvement}

\section{ACE - ACCEPTANCE, CHOICE AND EMPOWERMENT (ACE) FOR PRE -DIALYSIS PATIENTS: A PEER EDUCATOR BASED COMMUNITY: A PROJECT THAT AIMS TO IMPROVE CHOICE AND QUALITY OF LIFE FOR PATIENTS WITH KIDNEY FAILURE}

${ }^{1}$ Neerja Jain*, ${ }^{2}$ Jyoti Baharani, on behalf of the ACE Project team, ${ }^{2}$ Annette Dodds, ${ }^{1}$ Maz Ali, ${ }^{1}$ Peter Storey. ' Kidney Research UK; ${ }^{2}$ Birmingham Heartlands Hospital, University Hospital Birmingham

\subsection{6/leader-2018-FMLM.101}

Aims The recently released NICE guidance states that patient choice is at heart of dialysis treatment acceptance but most patients do not feel empowered enough to make this choice. Through innovative leadership and management, we sought to improve patients' acceptance of their kidney failure diagnosis and to empower them, regarding their dialysis treatment choices.

Methods Kidney Research UK has led a pilot project using its evidence based multi-award-winning Peer Educator (PE) model, involving trained and accredited volunteers who have a natural empathy with the patient group through language, culture, religion and healthcare experience.

$10 \mathrm{PE}$ volunteers were recruited with 9 remaining engaged during the 17 month pilot project, all of them with personal experience of renal replacement therapy.In partnership with patients and clinical staff (a consultant and 2-3 senior nurses were involved), they worked collaboratively to facilitate acceptance and decision making.

The project had a four- pronged approach:

- Visiting patients at home with the Kidney Nurse Specialist.

- Meeting patients in clinic,

- Phoning patients (if requested) post visit/clinic

- Organising Asian specific patient information days (these were later broadened to include all ethnic groups).

Results

- 14 Home visits undertaken. Patient X: 'I was very grateful that the nurse and PE took time to provide me with information. I was scared at first but was really happy that the PE spoke to me in my own language and explained the dialysis to me as she has been through it'

- 5 patient information days attended by 50 patients and 44 family members. In the past, the number of Asian patients attending such events had been poor, averaging just one/event so the bespoke events attracted almost $10 \mathrm{x}$ as many! 'It gave me a helpful insight into the reality of dialysis.' Feedback scores have been overwhelmingly positive and all patients said the project had helped them make a decision about their dialysis treatment

- 44 patients seen in clinic:E.g. 1 patient who found speaking with a PE, very cathartic - he cried but said it helped him to accept his diagnosis - finally.

- One key senior staff member described their change of attitude from initially feeling negative, cynical and anxious about the project based on previous experience of peer support, to subsequently feeling very passionate and felt it was essential to continue this service improvement!

- All the volunteer PEs expressed a commitment to continue to volunteer despite the formal end of the pilot project.

Conclusion This innovative pilot project utilising the Peer Educator approach in a novel way has, in a very short space of time, proved to be impactful and had very positive 'buy-in' from patients, staff and the PE volunteers. We believe some of the success is attributed to the ethos of involving and empowering patients, working in collaboration with renal health care professionals for the ultimate benefit of new renal patients. That is, through an innovative managerial approach. The project leadership team are highly committed and passionate about sustaining this work and helping spread to other units that would benefit from this initiative.

The project has achieved its aims of pilot testing a method to help with patient acceptance, choice and empowerment 
(ACE). The knowledge gained from this pilot shows that it is feasible to recruit, train and utilise the unique skills of patients as volunteers to support other (newly diagnosed) patients to accept their chronic, life changing condition, make informed choices about their treatment while empowering them.

We have the proof of principle that PEs can meet the needs of renal units, working within the NHS Trust volunteer/ governance framework. This initiative is low cost, demonstrating new ways of working and managing, empowering patients and utilizing non -clinical workforce through effective leadership.

\section{IMPROVING HEALTH OUTCOMES BY A SOUTH LONDON LOOKED AFTER CHILDREN'S (LAC) TEAM}

Stacy John-Legere*, Abby Sinclair. Evelina London, Guys and St. Thomas NHS Foundation Trust, UK

\subsection{6/leader-2018-FMLM.102}

Context There is an obligation on the local authority and its health partners to ensure these statutory health assessments take place in accordance with the timescales set. The looked after children's health team delivers this responsibility on behalf the local authority. A significant portion live outside the borough boundaries. The multidisciplinary team comprises specialist nurses, community paediatricians and administrative team.

Issue Timely and thorough health assessments are vehicles for identifying and acting upon previously unmet health needs in this vulnerable population.

Assessment of issue and analysis of its causes The Change Management Framework

The ADKAR ${ }^{\circledR}$ model of change was utilised. The team were aware of the need for change.The trust executive and management had a desire to support and participate in the change. We utilised the knowledge of the core team and additional expertise. We have also established a system of reinforcement to sustain the change.

The strategy:

To deliver on the changes needed a clear leadership strategy was in place.

- It was purpose driven

- Previous inspection reports, user feedback and performance indicators were used

- Clear and constant two-way communication was employed

o weekly performance huddles, and

- Investment in people and infrastructure occurred

$\circ$ job plans developed, and increased training and development opportunities given

- We demonstrated persistence to see the changes through

- Improvements were shared on the ground at team meetings, at directorate level; and at Trust executive level. The changes were shared with the Service Improvement Board, the CCG Safeguarding executive and the local authority.

Measurement of improvement and impact:

- In March 2018 100\% of children accessing initial assessments were seen within 20 working days from referral; the graph (figure 1) shows the overall trend of improvement

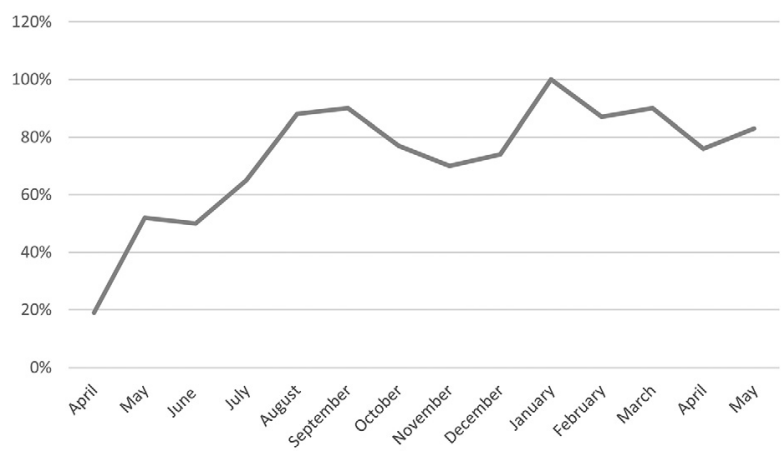

Abstract 104 Figure 1\% of children accessing initial assessments within 20 working days of referral- April 2017 to May 2018

- There is now knowledge of where all LAC are in the pathway, including those that are being seen out of borough and those that are in secure accommodation

- Improvement in staff morale measured by a fully established team, reduction in staff turnover rate and sickness levels

Impact The next steps are:

- Sustaining cultural and process changes in both the clinical and administrative team

- Continue to work with social care to improve the referral process

- Ensuring systematic oversight of the completion of health recommendations arising from assessment.

\section{DOCTOR (NOUN, LATIN), TO TEACH; TRANSFORMING A WORKING WEEK INTO A TEACHING WEEK}

${ }^{1}$ Ahmed Chilmeran*, ${ }^{2}$ Alexandros Rampotas, ${ }^{1}$ Mustafa Agbna, 'Sathianathan Panthakalam. ${ }^{1}$ East Sussex Healthcare NHS Trust, UK; ${ }^{2}$ Oxford Health NHS Foundation Trust, UK

\subsection{6/leader-2018-FMLM.103}

One of the prerequisites to successfully complete Core Medical Training in the UK is to acquire the MRCP title by passing the Practical Assessment of Clinical Examination Skills (PACES). Due to work pressures and time limitations, little teaching had been taking place locally at Eastbourne District General Hospital (EDGH). Prior to this, PACES teaching was mainly delivered externally by expensive courses, online resources and books. Given the plethora of patients in a familiar environment of an NHS hospital, we established that this provided an ideal combination for PACES teaching.

We started a team of PACES positive trainees who were interested in teaching; this team would deliver bedside PACES teaching at least twice a week. A weekly timetable with specified PACES teaching was created (e.g table 1). This included some specialised clinics that were consultant led (rheumatology and ophthalmology) aimed at providing consultant led teaching to PACES candidates. One PACES candidate was allocated on each clinic day providing 1-to-1 focused teaching, while the bedside sessions were open for everyone. A WhatsApp group was also created consisting of all candidates preparing for PACES and instructors, which encouraged all members to share interesting cases deemed good for PACES teaching and also facilitated organising unscheduled teaching. 